\title{
Avaliação do Potencial Eólico em uma Região do Sul do Brasil
}

\author{
Evaluation of Wind Potential in a Region of Southern Brazil
}

\begin{abstract}
${ }^{1}$ Alisson Nascimento, ${ }^{1}$ Silvana Maldaner, ${ }^{1}$ Vinicius Maran, ${ }^{2}$ Gervásio Annes Degrazia, ${ }^{2}$ Débora Regina Roberti, ${ }^{3}$ Virnei Silva Moreira, ${ }^{2}$ Marcelo Bortoluzzi Diaz, ${ }^{2}$ Michel Baptistella Stefanello e ${ }^{4}$ Umberto Rizza
\end{abstract}

${ }^{1}$ Universidade Federal de Santa Maria - campus Cachoeira do Sul

${ }^{2}$ Universidade Federal de Santa Maria

${ }^{3}$ Departamento de Engenharia - UNIPAMPA - Campus Itaqui

${ }^{4}$ ISAC-CNR - Itália

\section{Resumo}

O conhecimento do comportamento do vento desempenha um papel fundamental na produção de energia eólica, em ventilação de ambientes e na qualidade do ar. Assim, neste estudo o comportamento da velocidade do vento em Cachoeira do Sul (RS) é analisado. Dados de velocidade do vento medidos por um anemômetro sônico são empregados para estimar o potencial de geração de energia no período de 2010 à 2014. Uma das metodologias empregadas para o estudo do vento é a análise estatística utilizando funções de densidade de probabilidade. Existem diversos modelos de distribuição de probabilidade na literatura para séries temporais de dados. Para dados de vento a distribuição mais utilizada é a função de Weibull. Esta distribuição é considerada a mais adequada para caracterização do vento e é aplicada também na análise de dados de chuvas, índice de claridade, previsão do nível de água dentre outras aplicações. Assim, o objetivo do presente estudo é obter estimativas preliminares do potencial eólico de Cachoeira do Sul no RS empregando a distribuição de probabilidade de Weibull para estimar a potência do vento. Os resultados mostram que a potência do vento está abaixo de $500 \mathrm{~W} / \mathrm{m}^{2}$ (em $50 \mathrm{~m}$ ), o que indica baixa potencial eólico.

Palavras-chave: Potencial eólico, velocidade do vento, distribuição de probabilidade de Weibull.

\section{Abstract}

Knowledge of wind behavior plays a key role in the production of wind energy, in ambient ventilation and in air quality. In this study the wind speed behavior in Cachoeira do Sul (RS) is analyzed. Wind speed data was measured by a sonic anemometer and it was used to estimate the potential for power generation in the period from 2010 to 2014. One of the methodologies used for the study of wind was the statistical analysis using functions of probability density. There are several models of probability distribution in the literature for time series of data. For wind data, the most commonly used distribution is the Weibull function. This distribution is considered to be the most adequate for wind characterization and is also applied in the analysis of rainfall data, clarity index, water level prediction, among other applications. Thus, the objective of the present study is to obtain preliminary estimates of the wind potential of Cachoeira do Sul (RS) using the Weibull probability distribution to estimate the wind power. The results show that wind power is below $500 \mathrm{~W} / \mathrm{m}^{2}$ (in $50 \mathrm{~m}$ ) which indicates low wind potential.

Keywords: Wind potential, wind speed, Weibull probability distribution. 


\section{Introdução}

A demanda mundial de energia associada à diminuição dos combustíveis fósseis tem gerado uma corrida à geração de energias através de fontes renováveis. As fontes de energia renováveis como a solar, a biomassa e a eólica têm ganho destaque no cenário internacional.

No Brasil cerca de $60,97 \%$ da produção de eletricidade está associada à fontes hídricas, outros $26,7 \%$ provenientes de termoelétricas e 7,3\% de centrais geradoras eólica (ANEEL. Agência Nacional de Energia Elétrica, 2017). O percentual associado a produção eólica parece baixo, porém de 2007 a 2017 ocorreu um aumento de mais de $6 \%$ neste setor.

O baixo crescimento desta forma de energia está diretamente relacionada ao pouco conhecimento do campo de velocidade do vento e aos altos custos operacionais de instalação.

As primeiras medições de velocidade do vento no Brasil com anemômetros são da década de 1990 (DALMAZ, 2007). Hoje, o número de estações meteorológicas é pequeno, embora em crescimento.

$\mathrm{Na}$ literatura existem estudos sobre o potencial eólico de algumas regiões do nordeste e principalmente do litoral brasileiro. Considerando o país como um todo, Amarante (2001) afirma que o Brasil possui um potencial eólico da ordem de 143,47GW, considerando apenas regiões com velocidade média anual acima de $7 \mathrm{~m} / \mathrm{s}$, porém estas velocidades são encontradas em apenas $0,8 \%$ do território nacional.

Desta forma, neste trabalho o objetivo é investigar o comportamento do campo de vento em Cachoeira do Sul (RS-Brasil) e assim obter estimativas preliminares do potencial desta região empregando uma distribuição de probabilidade para estimar a potência do vento.

\section{Materiais e métodos}

Nesta seção serão apresentados os materiais e a metodologia utilizados neste estudo.

\subsection{Localização do sítio e coleta dos dados meteorológicos}

Os dados meteorológicos empregados neste estudo são provenientes da Rede Sul Brasileira de Fluxos Superficiais e Mudanças Climáticas (SULFLUX) e foram cedidos pelo grupo de meteorologia da Universidade Federal de Santa Maria - campus sede.

Os dados de velocidade do vento foram coletados por uma torre micrometeorológica situada em Cachoeira do Sul (lat. -30.2771 long. - 53.1479) no Rio Grande do Sul, Brasil. Cachoeira do Sul situa-se a 40,5m de altitude e está localizada na depressão central do estado. Os dados foram coletados à $3 m$ da superfície, a uma frequência de $10 \mathrm{~Hz}$ sobre uma plantação de arroz irrigado por inundação (SENA, 2011). Médias de bloco de trinta em trinta minutos foram utilizadas para facilitar a análise.

\subsection{Extrapolação da velocidade do vento para níveis superiores}

Para avaliar o potencial eólico de uma região, é observada a velocidade do vento à $50 \mathrm{~m}$. Desta forma, para extrapolar a velocidade do vento para a altura de $50 \mathrm{~m}$ a expressão da lei de potência para o vento (1) é empregada:

$$
v=v_{a}\left(z / z_{a}\right)^{\alpha}
$$

Na equação $1, z_{a}$ é a altura do anemômetro e $v_{a}$ é a velocidade do vento médio neste nível.

\subsection{Função densidade de probabilidade de Weibull}

A distribuição de Weibull pode ser escrita pela sua função de densidade de probabilidade $f(v)(2)$ :

$$
f(v)=\frac{k}{c}\left(\frac{v}{c}\right)^{k-1} \exp \left(-\left(\frac{v}{c}\right)^{k}\right)
$$

e pela função de distribuição acumulada $F(v)$ (CHANG, 2011; Pishgar-Komleh e Sefeedpari., 2015) 3:

$$
F(v)=1-\exp \left[-\left(\frac{v}{c}\right)^{k}\right]
$$

Na equação 3, $v$ é a velocidade do vento, $c$ é um parâmetro de escala que possui a mesma unidade de $v$ e $k$ é o parâmetro de forma (KWON, 2010). Os parâmetros $c$ e $k$ são calculados para cada série temporal de dados de vento e existem diversas formas de obtê-los. Neste estudo foi utilizado o método empírico, onde o parâmetro $k$ é estimado pela equação (4): 


$$
k=\left(\frac{\sigma}{\bar{v}}\right)^{-} 1,086
$$

Na equação (4) $\sigma$ é o desvio padrão de velocidade e $\bar{v}$ é a velocidade média do módulo do vento do período considerado. O parâmetro de escala $c$ é determinado pela expressão (5):

$$
c=\frac{\bar{v}}{\Gamma\left(1+\frac{1}{k}\right)}
$$

A partir do valor de $c$ e $k$ determina-se a densidade de energia do vento e a partir deste valor é possível verificar se uma região possui um regime de vento adequado ou não para a geração de energia eólica. A energia eólica disponível é definida pelo fluxo de energia cinética e é calculada através da equação (6):

$$
E(v)=\frac{1}{2} \rho v^{3} A[W]
$$

Na equação (6), $v$ é a velocidade do vento e $\rho$ é a densidade do ar (KWON, 2010; TAR, 2008; RAMÍREZ, 2005). Normalizando a equação 6 pela área, obtém-se (7):

$$
\frac{E(v) f(v)}{A}=\frac{1}{2} \rho v^{3} f(v)\left[W s / m^{3}\right]
$$

Integrando a equação (7) para todo o período de estudo encontra-se a densidade média de energia eólica em $W / \mathrm{m}^{2}$ no período de estudo $T$ ou potência do vento, obtém-se (equação 8):

$$
\bar{P}=\frac{1}{2} \rho \Gamma\left(1+\frac{3}{k}\right) T\left[W / m^{2}\right]
$$

Para calcular a densidade média de energia para o nível de $50 m$, JUSTUS (1976) afirmam que quando o parâmetro $c$ e $k$ de Weibull são conhecidos em uma determinada altura (altura do anemômetro), os valores destes parâmetros para qualquer outra altura podem ser obtidos pelas equações (9) e (10):

$$
\begin{gathered}
\frac{k 2}{k 1}=\frac{\left[1-0,0881 \ln \left(\frac{1}{10}\right)\right]}{\left[1-0,0881 \ln \left(\frac{z_{2}}{10}\right)\right]} \\
\frac{C_{2}}{C_{1}}=\left(\frac{z_{2}}{z_{1}}\right)^{n}
\end{gathered}
$$

com $n$ dado por (11):

$$
n=\frac{\left(0,37-0,0881 \ln \left(c_{1}\right)\right)}{\left[1-0,0881 \ln \left(\frac{z_{1}}{10}\right)\right]}
$$

onde $z_{1}$ e $z_{2}$ estão em metros e o índice 1 representa os valores associados com a altura do anemômetro e o índice 2 uma altura qualquer (JUSTUS, 1976).

\section{Resultados}

A tabela 1 apresenta uma descrição estatística dos dados de vento medidos em Cachoeira do Sul no período de janeiro de 2010 à dezembro de 2014. Nesta tabela a velocidade média mensal e a velocidade máxima são apresentadas por ano/mês de estudo. Pode-se observar que as velocidade médias estão em torno de $3 \mathrm{~m} / \mathrm{s}$, o que é um indicativo de um regime de vento de baixa velocidade do ponto de vista de engenharia do vento.

A partir dos parâmtros de Weibull obtidos pelo método empírico para 3 e $50 \mathrm{~m}$ e pela metodologia de JUSTUS (1976) para $50 \mathrm{~m}$, a densidade de energia do vento é calculada empregando a equação (8). 
Tabela 1: Características do vento.

\begin{tabular}{lllllll}
\hline Ano & & $\mathbf{2 0 1 0}$ & $\mathbf{2 0 1 1}$ & $\mathbf{2 0 1 2}$ & $\mathbf{2 0 1 3}$ & $\mathbf{2 0 1 4}$ \\
\hline \multirow{2}{*}{ Janeiro } & $v_{\max }$ & 7,99 & 7,56 & 6,03 & 6,75 & 7,66 \\
& $\bar{v}$ & 3,10 & 1,86 & 2,50 & 1,93 & 1,96 \\
Fevereiro & $v_{\max }$ & - & 5,87 & 5,79 & 6,32 & 7,10 \\
& $\bar{v}$ & - & 1,81 & 1,51 & 1,87 & 1,87 \\
Março & $v_{\max }$ & - & 7,17 & 6,34 & 5,94 & 5,36 \\
& $\bar{v}$ & - & 1,93 & 1,41 & 1,79 & 1,54 \\
Abril & $v_{\max }$ & 1,62 & 5,24 & 6,58 & 4,73 & 7,76 \\
& $\bar{v}$ & 0,88 & 1,38 & 1,49 & 1,34 & 1,79 \\
Maio & $v_{\max }$ & 7,02 & 5,93 & 6,66 & 7,79 & 6,49 \\
& $\bar{v}$ & 2,15 & 1,91 & 1,57 & 1,61 & 1,51 \\
Junho & $v_{\max }$ & 7,86 & 7,13 & 7,82 & 6,69 & 6,45 \\
& $\bar{v}$ & 2,18 & 2,32 & 1,70 & 1,61 & 1,75 \\
Julho & $v_{\max }$ & 8,28 & 7,84 & 7,40 & 6,42 & 6,91 \\
& $\bar{v}$ & 2,54 & 1,93 & 1,76 & 1,75 & 2,19 \\
Agosto & $v_{\max }$ & 11,35 & 11,81 & 6,82 & 6,68 & 7,97 \\
& $\bar{v}$ & 2,25 & 3,33 & 2,62 & 2,27 & 1,77 \\
Setembro & $v_{\max }$ & 9,85 & 8,17 & 14,66 & 9,59 & 7,88 \\
& $\bar{v}$ & 3,00 & 2,89 & 3,32 & 2,83 & 2,58 \\
\multirow{2}{*}{ Outubro } & $v_{\max }$ & 12,61 & 8,66 & 13,53 & 8,56 & 9,26 \\
& $\bar{v}$ & 2,46 & 2,86 & 3,30 & 2,89 & 3,02 \\
Novembro & $v_{\max }$ & 7,26 & 7,10 & 7,65 & 9,06 & 8,10 \\
\multirow{2}{*}{ Dezembro } & $\bar{v}$ & 2,57 & 2,66 & 2,76 & 3,46 & 2,58 \\
& $v_{\max }$ & 8,80 & 6,21 & 8,83 & 7,71 & 11,17 \\
\hline & $\bar{v}$ & 2,99 & 2,21 & 2,17 & 2,75 & 2,34 \\
\hline
\end{tabular}

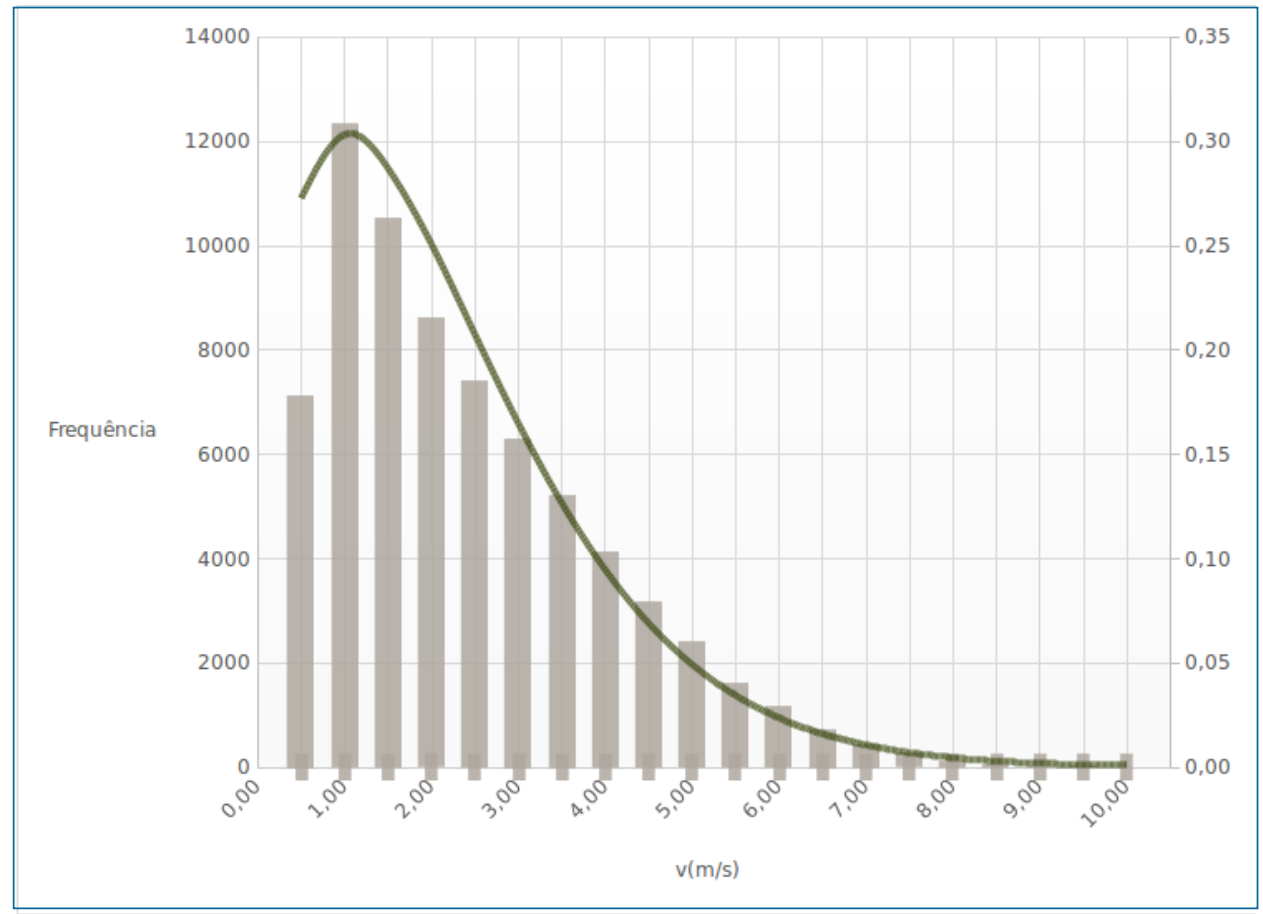

Figura 1: Frequência de ocorrência da velocidade do vento.

Os valores mensais para a potência do vento no período de estudo são apresentados na tabela 2 . O valor máximo da potência obtido para $50 m(p 2)$ foi de $287 \frac{\mathrm{W}}{\mathrm{m}^{2}}$ pelo método de JUSTUS (1976) e $189,12 \frac{\mathrm{W}}{\mathrm{m}^{2}}$ pelo método empirico ( $p 3$ ). Enquanto que para a superfície o valor máximo foi de $59 \frac{W}{m^{2}}$, apresentando um valor médio de menos de $20 \frac{W}{m^{2}}$ (em $3 m$ de altura $(p 1)$ ) de 2010 à 2014 . 
Tabela 2: Valores mensais para a potência do vento à $3 m(p 1), 50 m(p 2)$ e método empírico $(p 3)$.

\begin{tabular}{|c|c|c|c|c|c|c|c|c|c|}
\hline & & $p_{1}$ & $p_{2}$ & $p_{3}$ & & & $p_{1}$ & $p_{2}$ & $p_{3}$ \\
\hline \multirow[t]{12}{*}{2010} & Janeiro & 35.91 & 207.05 & 115.05 & \multirow[t]{6}{*}{2012} & Julho & 11.63 & 76.94 & 37.25 \\
\hline & Fevereiro & - & - & - & & Agosto & 20.96 & 137.45 & 67.17 \\
\hline & Março & - & - & - & & Setembro & 59.02 & 287.05 & 189.12 \\
\hline & Abril & 0.64 & 9.61 & 2.05 & & Outubro & 58.43 & 284.34 & 187.21 \\
\hline & Maio & 14.62 & 98.96 & 46.84 & & Novembro & 28.31 & 168.37 & 90.72 \\
\hline & Junho & 16.39 & 106.73 & 52.52 & & Dezembro & 15.52 & 103.13 & 49.74 \\
\hline & Julho & 20.95 & 134.64 & 67.12 & \multirow[t]{12}{*}{2013} & Janeiro & 10.10 & 75.13 & 32.36 \\
\hline & Agosto & 19.25 & 119.24 & 61.69 & & Fevereiro & 9.71 & 71.89 & 31.13 \\
\hline & Setembro & 42.43 & 223.47 & 135.96 & & Março & 9.32 & 68.40 & 29.86 \\
\hline & Outubro & 31.37 & 166.58 & 100.53 & & Abril & 5.05 & 40.34 & 16.18 \\
\hline & Novembro & 21.32 & 136.95 & 68.31 & & Maio & 8.52 & 60.88 & 27.31 \\
\hline & Dezembro & 34.14 & 197.02 & 109.39 & & Junho & 8.46 & 60.60 & 27.10 \\
\hline \multirow[t]{12}{*}{2011} & Janeiro & 10.18 & 73.64 & 32.61 & & Julho & 9.55 & 68.35 & 30.60 \\
\hline & Fevereiro & 8.52 & 65.47 & 27.29 & & Agosto & 16.13 & 108.24 & 51.67 \\
\hline & Março & 10.58 & 76.93 & 33.89 & & Setembro & 30.00 & 176.76 & 96.14 \\
\hline & Abril & 5.38 & 42.63 & 17.22 & & Outubro & 35.05 & 195.80 & 112.32 \\
\hline & Maio & 11.27 & 79.36 & 36.12 & & Novembro & 47.80 & 261.00 & 153.16 \\
\hline & Junho & 22.35 & 132.23 & 71.62 & & Dezembro & 28.42 & 168.36 & 91.07 \\
\hline & Julho & 14.92 & 93.75 & 47.81 & \multirow[t]{12}{*}{2014} & Janeiro & 10.98 & 79.36 & 35.17 \\
\hline & Agosto & 52.92 & 269.97 & 169.57 & & Fevereiro & 12.44 & 82.94 & 39.86 \\
\hline & Setembro & 38.12 & 205.34 & 122.16 & & Março & 6.26 & 49.71 & 20.05 \\
\hline & Outubro & 29.50 & 176.38 & 94.54 & & Abril & 10.90 & 74.88 & 34.93 \\
\hline & Novembro & 26.71 & 159.34 & 85.58 & & Maio & 7.79 & 55.65 & 24.95 \\
\hline & Dezembro & 17.01 & 109.72 & 54.49 & & Junho & 9.63 & 68.77 & 30.86 \\
\hline \multirow[t]{6}{*}{2012} & Janeiro & 20.82 & 132.99 & 66.72 & & Julho & 16.19 & 106.13 & 51.88 \\
\hline & Fevereiro & 4.87 & 42.55 & 15.62 & & Agosto & 11.11 & 75.10 & 35.61 \\
\hline & Março & 5.01 & 41.45 & 16.07 & & Setembro & 24.52 & 148.81 & 78.57 \\
\hline & Abril & 5.94 & 47.29 & 19.05 & & Outubro & 37.92 & 210.19 & 121.51 \\
\hline & Maio & 9.16 & 62.68 & 29.36 & & Novembro & 26.59 & 155.88 & 85.21 \\
\hline & Junho & 8.96 & 64.86 & 28.70 & & Dezembro & 18.36 & 118.83 & 58.83 \\
\hline
\end{tabular}

A figura 1 apresenta o histograma de velocidade do vento médio com as respectivas frequências de ocorrência da velocidade vento e a função ajustada de Weibull ajustada ao conjunto de dados para janeiro de 2010 à dezembro de 2014.

Nesta figura, pode-se observar claramente que a maior frequência de ocorreria da velocidade do vento está entre $1 \mathrm{e} 1,5 \mathrm{~m} / \mathrm{s}$, reforçando o resultado de que em Cachoeira do Sul os ventos são de baixa magnitude. Além disso, os valores de $k$ e $c$ encontrados para todo o período(2010-2014) foram 1,41 e 2,43 respectivamente.

\section{Conclusão}

Neste trabalho o comportamento da velocidade do vento em Cachoeira do Sul foi analisado. Dados de vento coletados por um anemômetro sônico a $3 m$ de altura foram empregados para analisar o potencial eólico do local.

Os resultados mostraram que o local possui velocidade médias em torno de $2,5 \frac{\mathrm{m}}{\mathrm{s}}$ caracterizando um regime de vento de baixa magnitude. A potência média do vento para uma altura de $3 \mathrm{~m}$ foi da ordem de aproximadamente $19 \frac{\mathrm{W}}{\mathrm{m}^{2}}$, para $50 \mathrm{~m}$ empregando a metodologia de JUSTUS (1976) obteve-se $287,05 \frac{\mathrm{W}}{\mathrm{m}^{2}}$, enquanto que a utilização do método empírico resultou em $189,12 \frac{\mathrm{W}}{\mathrm{m}^{2}}$.

Oberva-se que o uso da metodologia empírica implica em resultados inferiores de densidade de energia. Tomando por base os trabalhos de Johansson e Burnham (1993) para que uma região seja considerada com potencial para geração de energia eólica, sua densidade de energia deve ser maior ou igual a $500 \frac{\mathrm{W}}{\mathrm{m}^{2}}$ a uma altura de $50 \mathrm{~m}$.

Desta forma, conclui-se que a cidade de Cachoeira do Sul não é adequada para projetos eólicos que envolvam geradores de eixo horizontal de grande porte. O estudo foi importante, pois através do conhecimento da magnitude do vento médio conclui-se que o local de estudo não é adequado para geradores de eixo horizontal.

Entretanto, este conhecimento permitirá planejar geradores eólicos de eixo vertical, os quais funcionam em baixas velocidades 
do vento, além de permitir a realização de planejamentos nas áreas de ventilação natural de edifícios e na área agrícola para palnejamento de aplicação de defensivos e irrigação.

\section{Referências}

Amarante, O. A. e. (2001). Atlas do potencial eólico brasileiro.

ANEEL. Agência Nacional de Energia Elétrica (2017). Big - banco de informações de geração. URL http: / /www2 . aneel . gov.br/aplicacoes/capacidadebrasil/capacidadebrasil.cfm.

CHANG, T. P. (2011). Performance comparison of six numerical methods in estimating weibull parameters for wind energy application. Applied Energy, 88, 272-282.

DALMAZ, A. (2007). Estudo do potencial eólico e previsão de ventos para geração de eletricidade em santa catarina. Doutorado em engenharia mecânica, Universidade Federal de Santa Catarina, Florianópolis.

Johansson, T. B., Burnham, L. (1993). Renewable energy: sources for fuels and electricity. Island press.

JUSTUS, A., C. G.; MIKHAIL (1976). Height variation of wind speed and wind distributions statistics. Geophysical Research Letters, 3, 261-264.

KWON, S. D. (2010). Uncertainty analysis of wind energy potential assessment. Applied Energy, 87, 856-86.

Pishgar-Komleh, A. K., S. H., Sefeedpari., P. (2015). Wind speed and power density analysis based on weibull and rayleigh distributions (a case study: Firouzkooh county of iran). Renewable and Sustainable Energy Reviews, 42, 313-322.

RAMÍREZ, J. A., Penélope; CARTA (2005). Influence of the data sampling interval in the estimation of the parameters of the weibull wind speed probability density distribution: a case study. Energy Conversion and Management, 46, 2419-2438.

SENA, J. e. a. (2011). Análise das componentes do balanço energético numa lavoura de arroz irrigado. Ciência e Natura, ed. especial., 143-146.

TAR, K. (2008). Some statistical characteristics of monthly average wind speed at various heights. Renewable and Sustainable Energy Reviews, 12, 1712-1724.

Alisson Nascimento

Universidade Federal de Santa Maria - campus Cachoeira do Sul E-mail: alisson.300@hotmail.com

Silvana Maldaner

Vinicius Maran

Gervásio Annes Degrazia

Débora Regina Roberti
Universidade Federal de Santa Maria - campus Cachoeira do Sul E-mail: silvana.maldaner@gmail.com

Universidade Federal de Santa Maria - campus Cachoeira do Sul E-mail: viniciusmaran@gmail.com

Universidade Federal de Santa Maria E-mail: gervasiodegrazia@gmail.com

Universidade Federal de Santa Maria E-mail: debora@ufsm.br

\section{Virnei Silva Moreira}

Departamento de Engenharia - UNIPAMPA - Campus Itaqui 


\section{Marcelo Bortoluzzi Diaz}

Universidade Federal de Santa Maria E-mail: marbdiaz@gmail.com

Michel Baptistella Stefanello

Universidade Federal de Santa Maria E-mail: michelstefanello@gmail.com

\section{Umberto Rizza}

ISAC-CNR - Itália

E-mail: izza@le.isac.cnr.it 